\title{
Singular radial solutions for the Lin-Ni-Takagi equation
}

\section{Casteras, Jean-Baptiste}

2020-09-13

Casteras , J-B \& Földes , J 2020 , ' Singular radial solutions for the Lin-Ni-Takagi equation ' , Calculus of Variations and Partial Differential Equations, vol. 59 , no. 5 , 168 . https://doi.org/10.1007/s00526-020-0

http://hdl.handle.net/10138/321645

https://doi.org/10.1007/s00526-020-01824-3

publishedVersion

Downloaded from Helda, University of Helsinki institutional repository.

This is an electronic reprint of the original article.

This reprint may differ from the original in pagination and typographic detail.

Please cite the original version. 


\title{
Singular radial solutions for the Lin-Ni-Takagi equation
}

\author{
Jean-Baptiste Casteras ${ }^{1}$. Juraj Földes ${ }^{2}$
}

Received: 1 May 2020 / Accepted: 31 July 2020 / Published online: 13 September 2020

( ) Springer-Verlag GmbH Germany, part of Springer Nature 2020

\begin{abstract}
We study singular radially symmetric solution to the Lin-Ni-Takagi equation for a supercritical power non-linearity in dimension $N \geq 3$. It is shown that for any ball and any $k \geq 0$, there is a singular solution that satisfies Neumann boundary condition and oscillates at least $k$ times around the constant equilibrium. Moreover, we show that the Morse index of the singular solution is finite or infinite if the exponent is respectively larger or smaller than the Joseph-Lundgren exponent.
\end{abstract}

Mathematics Subject Classification 35B32 $\cdot 35 \mathrm{~B} 05 \cdot 35 \mathrm{~J} 15 \cdot 34 \mathrm{~B} 40$

\section{Introduction}

In the present paper we study singular solutions of the problem

$$
\left\{\begin{aligned}
-\Delta v+v=v^{p} & \text { in } B_{R} \backslash\{0\}, \\
v>0 & \text { in } B_{R} \backslash\{0\}, \\
\partial_{\nu} v=0 & \text { on } \partial B_{R},
\end{aligned}\right.
$$

where $B_{R} \subset \mathbb{R}^{N}, N \geq 3$ is a ball of radius $R>0$ centred at the origin. We show that for sufficiently large $p$ (super-critical), (1.1) possesses a radial solution with many oscillations. The importance of singular solutions stems from the fact that they are asymptotes to the bifurcation branches of regular solutions. Therefore, we investigate the Morse index of

Communicated by A. Malchiodi.

J. Földes is partly supported by the National Science Foundation under the Grant NSF-DMS-1816408.

Juraj Földes

foldes@virginia.edu

Jean-Baptiste Casteras

jeanbaptiste.casteras@gmail.com

1 Department of Mathematics and Statistics, University of Helsinki, P.O. Box 68, Pietari Kalmin katu 5, 00014 Helsinki, Finland

2 Department of Mathematics, University of Virginia, 322 Kerchof Hall, Charlottesville, VA 22904-4137, USA 
singular solutions, which indicates the oscillatory or non-oscillatory nature of the bifurcation branches.

The problem (1.1) arises as a particular case of the stationary Keller-Segel system which is a reaction-diffusion system modelling chemotaxis-oriented motion of cells toward higher or lower concentrations of chemicals. One can also derive (1.1) from the activator-inhibitor system proposed by Gierer and Meinhardt [14] under the assumption that one chemical diffuses much faster than the other one. Gierer-Meinhardt system was extensively studied during last decades (see for example [29] and references therein), since it is one of the first and simplest examples of the diffusion driven instability.

Equation (1.1) with sub-critical exponent on smooth domains has been intensively studied in the last decades. More precisely, consider

$$
\left\{\begin{aligned}
-\Delta v+\tilde{\lambda} v=v^{p} & \text { in } \Omega, \\
v>0 & \text { in } \Omega, \\
\partial_{\nu} v=0 & \text { on } \partial \Omega,
\end{aligned}\right.
$$

where $\Omega \subset \mathbb{R}^{N}, N \geq 3$, is an open smooth domain, $\tilde{\lambda}>0$ and $1 \leq p \leq 2^{*}-1=\frac{N+2}{N-2}$. In a series of seminal works [18,24,25], Lin, Ni, and Takagi proved the existence of families of solutions concentrating around one or more points. Specifically, they showed that, when $1<p<2^{*}-1$, then the least energy solution $u_{\tilde{\lambda}}$ to (1.2) satisfies

$$
u_{\tilde{\lambda}} \rightarrow \tilde{\lambda}^{\frac{1}{p-1}} U\left(\sqrt{\tilde{\lambda}}\left(x-x_{\tilde{\lambda}}\right)\right), \quad \text { as } \quad \tilde{\lambda} \rightarrow \infty
$$

where $x_{\tilde{\lambda}} \in \partial \Omega$ converges to the boundary point with the maximal mean curvature and the asymptotic profile $U$ is the unique positive radial solution to

$$
-\Delta U+U=U^{p}, \lim _{|x| \rightarrow \infty} U(y)=0, \quad \text { in } \mathbb{R}^{N} .
$$

Observe that the parameter $\tilde{\lambda}$ is related to the size of the domain. Indeed, let $v$ be for example a solution of (1.2) with $\Omega=B_{R}$. Then, by setting $u(x)=R^{\frac{2}{p-1}} v(R x)$, we see that $u$ satisfies

$$
-\Delta u+R^{2} u=u^{p}, \quad \text { in } B_{1} .
$$

We refer to $[2,7,17,20]$ and the references therein for construction and analysis of families of solutions concentrating on multiple points located either in the interior of $\Omega$ and/or at the boundary.

The position of spikes is more restricted if $p$ is critical, that is, if $p=2^{*}-1$. Then, it is possible to show the concentration/bubbling phenomena when $\lambda \rightarrow \infty$ with asymptotic profile being the standard bubble, that is, the unique (up to scaling and translations) solution to

$$
-\Delta U=U^{2^{*}-1}, \quad \text { in } \mathbb{R}^{N}
$$

When $p$ is critical and $N=3$ or $N \geq 7$, it has been proved that there is no solution bubbling at an interior point of the domain $[12,26]$. Moreover, in arbitrarily dimension, interior bubbling solutions can only exist if they are bubbling also at the boundary [27]. We refer to [9] for construction of families of bubbling solutions when $p$ approaches from below or above the critical one. We also very briefly point out that families of solutions concentrating on higher dimensional object have been obtained see for instance $[1,8]$ and the references therein. 
In the supercritical case $p>2^{*}-1$, there is another significant exponent found by Joseph and Lundgren [16]

$$
p_{J L}= \begin{cases}1+\frac{4}{N-4-2 \sqrt{N-1}}, & N \geq 11, \\ \infty, & 3 \leq N \leq 10,\end{cases}
$$

which is connected to the number of intersections of radial solutions, and therefore to the stability with respect to compactly supported perturbations. First bifurcation results in supercritical range were obtained for radial solutions solving (1.3) with Dirichlet boundary conditions, or more generally for

$$
\left\{\begin{array}{l}
U_{r r}+\frac{N-1}{r} U_{r}+\lambda g(U)=0,0<r<1, \\
U>0,0<r<1, \\
U^{\prime}(0)=U(1)=0,
\end{array}\right.
$$

see $[6,10,15,16,21,22]$. If $g(U)=e^{U}$ or $g(U)=(1+U)^{p}$, Joseph and Lundgren in [16] (see also [11, Chapter 2] for a recent survey) showed that there is a curve of positive solutions to (1.4) starting from the trivial solution $U=0$ and $\lambda=0$. Moreover, they proved that this branch oscillates around a fixed value of $\lambda$ when $3 \leq N \leq 9$ in case $g(U)=e^{U}$ respectively $p_{S}<p<p_{J L}$ when $g(U)=(1+U)^{p}$ (see Theorem 1.1 below for more precise statement). On the other hand, the branch does not oscillate when $N \geq 10$ respectively $p \geq p_{J L}$. Let us point out that Gel'fand [13] was the first one who treated the case $N=3$ for $g(U)=e^{U}$.

In the literature, there are fewer results for (1.3) with Neumann conditions. The first reason might be that there are infinitely many branches with positive radial solutions, which are harder to analyze. The second reason might be practical, as in the Dirichlet case the radial solutions are stable at least in some parameter ranges, whereas in Neumann case, the radial solutions have large Morse index in the space of all (even non-radial) functions. Nevertheless, the bifurcation results were obtained by Miyamoto [23], who analyzed bifurcations of radial solution to (1.2) with respect to the parameter $\tilde{\lambda}$. To make the notation compatible with [23], note that after scaling $v$ we can rewrite (1.2) for $\Omega=B_{R}$ as

$$
\left\{\begin{array}{cl}
-\Delta v=\lambda\left(v^{p}-v\right) & \text { in } B_{R}, \\
v>0 & \text { in } B_{R}, \\
\partial_{\nu} v=0 & \text { on } \partial B_{R} .
\end{array}\right.
$$

Theorem 1.1 ([23]) Suppose that $p>2^{*}-1$. Let $\mathcal{S}$ denote the set of the regular, radial solutions of (1.5). Then

$$
\mathcal{S}=\mathcal{C}_{0} \cup \bigcup_{n=1}^{\infty} \mathcal{C}_{n},
$$

where $\mathcal{C}_{0}=\{\lambda, 1\}$. Moreover, since solutions are radial and $v^{\prime}(0)=0$, each $\mathcal{C}_{n}$ can be parametrized by $\gamma=v(0) \in(0, \infty)$, hence $\mathcal{C}_{n}=\left\{\left(\lambda_{n}(\gamma), v\left(\cdot, \gamma, \lambda_{n}(\gamma)\right)\right)\right\}$. Furthermore, $\gamma \mapsto \lambda_{n}(\gamma) \in C^{1}(0, \infty)$ and the following assertions hold :

(i) For each $n \geq 1, \lambda_{n}(1)=\bar{\lambda}_{n}$, where $\bar{\lambda}_{n}=\frac{\mu_{n}}{p-1}$ and $\mu_{n}$ is the $n$-th eigenvalue of the Laplacian with Neumann boundary condition.

(ii) For each $n \geq 1$, there exists $\lambda_{n}^{*}>0$ such that $\lambda_{n}(\gamma) \rightarrow \lambda_{n}^{*}$ as $\gamma \rightarrow \infty$.

(iii) If $p<p_{J L}$, then for each $n \geq 1, \lambda_{n}(\gamma)$ oscillates around $\lambda_{n}^{*}$ infinitely many times as $\gamma \rightarrow \infty$. More precisely, there exists a sequence $\left(\gamma_{m}^{n}\right)_{m}$ with $\gamma_{m}^{n} \rightarrow \infty$ as $m \rightarrow \infty$ such that $\lambda_{n}\left(\gamma_{m}^{n}\right)=\lambda_{n}^{*}$. 
(iv) For each $n \geq 1, \lambda_{n}(\gamma) \rightarrow \infty$ as $\gamma \rightarrow 0^{+}$.

(v) For each $\gamma \in(0, \infty), \lambda_{1}(\gamma)<\lambda_{2}(\gamma)<\ldots$..

As mentioned above, the parameter $\lambda$ is connected to the size of the domain, which is in many models fixed (see Keller-Segel system and chemotaxis). However, other constants such as diffusivity can change, and these, after scaling, are related to $p$. Hence, instead of changing the domain, that is, the parameter $\lambda$, we fix the domain and vary $p$. We recall bifurcation results in such case from [5]. Here and below $p_{i}^{r a d}$ denotes the $i$-th radial eigenvalue of the operator $-\Delta+I d$ in the ball $B_{R}:=\left\{x \in \mathbb{R}^{N}:|x|<R\right\}$ with Neumann boundary conditions.

Theorem 1.2 ([5]) For every $i \geq 2$, the trivial branch $(p, 1)$ of problem (1.1) has a bifurcation point at $\left(p_{i}^{\text {rad }}, 1\right)$. If $\mathcal{B}_{i} \subset \mathbb{R}^{2}$, parametrized by $(p, u(0))$, is the continuum that branches out of $\left(p_{i}^{r a d}, 1\right)$, then the following holds:

(i) The branches $\mathcal{B}_{i}$ are unbounded and do not intersect. Furthermore, near $\left(p_{i}^{\text {rad }}, 1\right), \mathcal{B}_{i}$ is a $C^{1}$-curve.

(ii) If $(p, A) \in \mathcal{B}_{i}$, then the corresponding solution $u_{p}$ satisfies $u_{p}>0$ in $B_{R}$.

(iii) Each branch consists of two connected components $\mathcal{B}_{i}^{-}:=\mathcal{B}_{i} \cap\{(p, A): A<1\}$ and $\mathcal{B}_{i}^{+}:=\mathcal{B}_{i} \cap\{(p, A): A>1\}$.

(iv) If $(p, A) \in \mathcal{B}_{i}$ then the corresponding $u_{p}-1$ has exactly $i-1$ zeros, $u_{p}^{\prime}$ has exactly $i$ zeros (including ones on the boundary and at the origin).

(v) The functions satisfying $u_{p}(0)<1$ are uniformly bounded in the $C^{1}$-norm.

Previously, by different techniques the lower branches $\mathcal{B}_{i}$ were presumably constructed in [4] and the first upper branch $\mathcal{B}_{2}$ by [28] when $N=3$.

The goal of this paper is to establish oscillatory results for upper branches as in Theorem (1.1) or as in the Dirichlet case. In the following, we will only be concerned with upper branches for $p>2^{*}-1$ and their asymptotics when $p$ gets large. Of course, in the finite range the branches can have only finitely many turns, and therefore large $p$ behaviour determines on oscillations or non-oscillation of branches. We focus on singular solutions, that are limit profiles of bifurcation branches as proved by Miyamoto in the following theorem.

Theorem 1.3 ([23]) Let $N \geq 3$ and $p>2^{*}-1$. There is a unique solution $U_{p}^{*}:=U^{*}$ to

$$
\begin{cases}-u^{\prime \prime}-\frac{N-1}{r} u^{\prime}+u=u^{p}, & \text { in } \mathbb{R}^{+}, \\ \lim _{r \rightarrow 0^{+}} r^{\theta} u(r)=A_{p, N}, & \text { in } \mathbb{R}^{+},\end{cases}
$$

where

$$
\theta=\frac{2}{p-1}, \text { and } A_{p, N}=[\theta(N-2-\theta)]^{\frac{1}{p-1}} .
$$

Moreover, $U^{*}$ attains infinitely many times the value 1 . Furthermore, if there are sequences $\left(\gamma_{n}\right)_{n}$ and $\left(p_{n}\right)_{n}$ with $\gamma_{n} \rightarrow \infty$ and $p_{n} \rightarrow p_{\infty}>2^{*}-1$, then $u_{\gamma_{n}, p_{n}} \rightarrow U_{p_{\infty}}^{*}$ in $C_{l o c}^{0}(0, \infty)$, where $u_{\gamma, p}$ is the solution to

$$
\left\{\begin{array}{l}
-u^{\prime \prime}-\frac{N-1}{r} u^{\prime}+u=u^{p}, \text { in } \mathbb{R}, \\
u(0)=\gamma, u^{\prime}(0)=0 .
\end{array}\right.
$$


Since $U^{*}$ attains infinitely many times the value 1 , there exists an increasing sequence $\left(R_{p}^{i}\right)_{i}$ such that $\left(U_{p}^{*}\right)^{\prime}\left(R_{p}^{i}\right)=0$, and therefore $U_{p}^{*}$ is a solution of (1.1) with $R$ replaced by $R_{p}^{i}$. However, if the size of the domain is fixed, then the existence of singular solution does not follow from Theorem 1.3, unless one is willing to change the equation (or more precisely $\lambda$ ) by scaling as in (1.5).

In our main result, we show that, for a fixed radius $R$ and any large integer $i>1$, we can find $p>2^{*}-1$ such that $R_{p}^{i}=R$. In other words, for any $R$ fixed, we are able to construct a singular solution to (1.1) having a prescribed number of intersections with 1 (and therefore a prescribed number of critical points). Since by Theorem 1.2 all solutions on $\mathcal{B}_{i}^{+}$ have exactly $i$ critical points, we believe that the limit point of $\mathcal{B}_{i}^{+}$is exactly the constructed singular solution with $i$ critical points.

Our theorem also complements the results proved by Lin and $\mathrm{Ni}$ [19] that, asserts that for any fixed $p>2^{*}-1$, there exists $R^{*}$ depending on $p$ and $N$ such that, for all $R<R^{*}$, equation (1.1) only admits constant solutions.

Theorem 1.4 Let $N \geq 3$ and $R>0$. Fix $\tilde{p}>2^{*}-1$ and let $U_{\tilde{p}}^{*}$ be the solution to (1.6). Let $i^{*}$ be the smallest integer such that $R_{\tilde{p}}^{i^{*}}>R$. Then, for any $i \geq i^{*}$, there exists $p^{i}>2^{*}-1$ such that

$$
R_{p^{i}}^{i}=R
$$

In particular, for any $i \geq i^{*}$, there exists $p^{i}>2^{*}-1$ such that equation (1.1) admits a singular radial solution $U$ satisfying

$$
\sharp\{r \in[0, R] \mid U(r)=1\}=i .
$$

We remark that an analogous result with $u^{p}$ replaced by $\lambda e^{u}$ (with $\lambda$ as a bifurcation parameter) been obtained by the authors and Bonheure in [3].

Next, we investigate the asymptotic behavior of the branch $\mathcal{B}_{i}^{+}$. The following theorem proved in [23] gives a strong indication that for each $i \geq 1$, the branch $\mathcal{B}_{i}^{+}$oscillates around $p^{i}$ (see Theorem 1.4) when $2^{*}-1<p^{i}<p_{J L}$. Fix $p>2^{*}-1$ and $\gamma_{0}$. We denote by $\left(r_{p, \gamma}^{i}\right)_{i}$, the increasing sequence of positive real numbers satisfying $u_{\gamma, p}^{\prime}\left(r_{p, \gamma}^{i}\right)=0$, where $u_{\gamma, p}$ is the unique solution to (1.7).

Theorem 1.5 [23, Theorem 6.1] Let $R>0, N \geq 11, i \geq i^{*}$, and $2^{*}-1<p^{i}<p_{J L}$. Then, there exist a sequence of initial data $\left(\gamma_{n}\right)_{n}$ and a sequence of positive integer $\left(j_{n}\right)_{n}$ such that $\gamma_{n} \rightarrow \infty$ and $r_{p^{i}, \gamma_{n}}^{j_{n}}=R$.

Note that since $j_{n}$ in general depends on $n$, one cannot conclude that the points $\left(p^{i}, \gamma_{n}\right)$ lie on $\mathcal{B}_{i}$. Also, without additional information one cannot combine Theorem 1.5 and Theorem 1.3 to prove Theorem 1.4 by limiting procedure. We remark that the oscillations and convergence of $\mathcal{B}_{i}$ was proved by authors and Bonheure in [3] for (1.1) with $v^{p}$ replaced by $\lambda e^{v}$. The proof in the present case is more involved and will be published separately.

A strong indication that branches oscillate when $p^{i}<p_{J L}$ and do not oscillate when $p^{i}>p_{J L}$ is provided by the radial Morse index of our singular solution. Recall that the Morse index of $v$ satisfying (1.1), denoted by $m(v)$, in the space of radial functions is the number of negative eigenvalues $\alpha$ counted with multiplicity of the following eigenvalue problem

$$
\left\{\begin{aligned}
-\Delta \phi+\phi-p u^{p-1} \phi=\alpha \phi & \text { in } B_{R} \backslash\{0\}, \\
\partial_{\nu} \phi=0 & \text { on } \partial B_{R}, \\
\phi \quad \text { is radially symmetric. } &
\end{aligned}\right.
$$


Note that each turn of the bifurcation branch increases the Morse index of solutions, thus finite or infinite Morse index of the limit (singular solution) suggest respectively non-oscillatory or oscillatory behaviour.

Proposition 1.6 Let $U_{p^{i}}^{*}$ be a solution to (1.6), where $p^{i}$ is as in Theorem 1.4. Then $m\left(U_{p^{i}}^{*}\right)<$ $\infty$ if $p^{i}>p_{J L}$, while $m\left(U_{p^{i}}^{*}\right)=\infty$ if $2^{*}-1<p^{i}<p_{J L}$.

Finally, we briefly sketch main ideas of the proofs. To prove Theorem 1.4, we follow the general framework used in [3]. Specifically, Theorem 1.4 is a consequence of continuity of the function $p \rightarrow R_{p}^{i}$ for all $i \in \mathbb{N}$ and

$$
R_{i}^{p} \rightarrow 0^{+} \text {as } p \rightarrow \infty \quad \text { for all } i \in \mathbb{N} \text {. }
$$

To establish of (1.8), as in [3], we obtain very precise estimates of $U_{p}^{*}$ in a neighbourhood of the origin. It is crucial to control the size of the neighbourhood with respect to parameter $p$. The proof is rather technical and requires very detailed information about solutions. Unlike in [3], our estimates cease to hold before the first intersection point with 1 that we denote $r_{p}$. At least heuristically $r_{p} \approx \frac{1}{\sqrt{p}}$ (in fact the upper bound can be made rigorous). Although we are not able to control the solution till $r_{p}$ we obtain estimates on the interval of comparable length $\left[0, \frac{\tilde{c}}{p}\right]$, where $\tilde{c}$ is sufficiently small constant. The key ingredient is the negativity of the higher order correction of $U_{p}^{*}$. Note that such estimate would not suffice in [3], however since our constant equilibrium (equal to 1 ) is independent of $p$, we could proceed.

Consequently, we prove that $\left(U_{p}^{*}\right)^{\prime}\left(\frac{\tilde{c}}{p}\right)$ converges to 0 when $p \rightarrow \infty$. Using the decay of an energy functional, we show that $U_{p}^{*}(r)$ stays very close to 1 for any $r \geq \frac{\tilde{c}}{p}$ and we are conclude by using the Sturm-Piccone theorem.

The continuity of the function $p \rightarrow R_{p}^{i}$ relies heavily on the uniqueness of $U_{p}^{*}$ and again the precise estimates at the origin on a controlled interval.

Proposition 1.6 containing the estimates on the Morse index of $U_{p}^{*}$ relies on the asymptotic behaviour of $U_{p}^{*}$ when $r \rightarrow 0$ and the Hardy's inequality.

\section{Proof of Theorem 1.4}

In this section, we prove Theorem 1.4. It will be a consequence of the continuity of the function $p \rightarrow R_{p}^{i}$, for all $i \in \mathbb{N}$ and the fact that

$$
R_{p}^{i} \rightarrow 0^{+}, \text {as } p \rightarrow \infty
$$

First, we prove that (2.1) holds true. In all the following, we denote by $U^{*}:=U_{p}^{*}$ the singular solution of (1.6). Before proceeding, let us give several definitions and recall some facts. We begin by introducing a change of variables which was already used in [23] to prove the existence of a singular solution.

Define

$$
\eta(\zeta)=A_{p, N}^{-1} r^{\theta} U^{*}(r)-1, \quad-\zeta=m^{-1} \ln r
$$

where $A_{p, N}$ and $\theta$ are defined in Theorem 1.3 and

$$
m=[\theta(N-2-\theta)]^{-\frac{1}{2}}
$$


It is easy to check that $\eta$ satisfies

$$
\left\{\begin{array}{l}
\eta^{\prime \prime}-\alpha \eta^{\prime}+(p-1) \eta=-(1+\eta)^{p}+1+p \eta+m^{2} e^{-2 m \zeta}(1+\eta), \text { on } \mathbb{R} \\
\lim _{\zeta \rightarrow \infty} \eta(\zeta)=0
\end{array}\right.
$$

where

$$
\alpha=m(N-2-2 \theta) \text {. }
$$

Next, we set $\tilde{\eta}=\eta-f$, where $f(\zeta)=D_{p} e^{-2 m \zeta}$ and $D_{p}=\frac{m^{2}}{4 m^{2}+2 \alpha m+(p-1)}$. Then, a straightforward computation shows that

$$
\tilde{\eta}^{\prime \prime}-\alpha \tilde{\eta}^{\prime}+(p-1) \tilde{\eta}=m^{2} e^{-2 m \zeta} \eta+\phi(\eta)=: \tilde{g},
$$

where

$$
\phi(\eta)=-\left((1+\eta)^{p}-1-p \eta\right) .
$$

We will also make intensive use of the following representation formula :

$$
\tilde{\eta}=\int_{\zeta}^{\infty} G_{N}(\sigma-\xi) \tilde{g}(\sigma) d \sigma
$$

where

$$
G_{N}(x)=\left\{\begin{array}{ll}
\frac{e^{-\frac{\alpha}{2} x}}{\beta} \sin (\beta x), & \text { if } p-1>(\alpha / 2)^{2} \\
\frac{e^{-\frac{\alpha}{2}} x}{\beta} \sinh (\beta x), & \text { if } p-1<(\alpha / 2)^{2}, \\
e^{-\frac{\alpha}{2} x} x, & \text { if } p-1=(\alpha / 2)^{2}
\end{array} \quad \text { for } x \geq 0, \quad G_{N}(x)=0, \text { if } x<0 .\right.
$$

and $\beta=\sqrt{\left|p-1-(\alpha / 2)^{2}\right|}$. Using that $\lim _{p \rightarrow \infty} \frac{1}{p-1}\left(\frac{\alpha}{2}\right)^{2}=\frac{N-2}{8}$ and $\frac{1}{p-1}\left(\frac{\alpha}{2}\right)^{2}=\frac{(8-2 \theta)^{2}}{8(8-\theta)}$ when $N=10$, we deduce that, for $p$ large enough, $p-1>\left(\frac{\alpha}{2}\right)^{2}$ if $N \leq 10$ and $p-1<\left(\frac{\alpha}{2}\right)^{2}$ if $N>10$.

We also define $w(r)=r^{\frac{N-1}{2}}\left(U^{*}(r)-1\right)$. By standard manipulations, one has

$$
w^{\prime \prime}+\left(\frac{\left(U^{*}\right)^{p}-U^{*}}{U^{*}-1}-\frac{(N-1)(N-3)}{4 r^{2}}\right) w=0 .
$$

The following asymptotics when $p \rightarrow+\infty$ of parameters are useful below

$$
\begin{aligned}
& \lim _{p \rightarrow \infty} \frac{\beta}{\sqrt{p}}=\sqrt{\left|1-\frac{N-2}{8}\right|} \text { if } N \neq 10, \quad \beta=\sqrt{\frac{3(p-1)-1}{4(p-1)-1}} \text { if } N=10, \\
& \lim _{p \rightarrow \infty} p \theta=2, \lim _{p \rightarrow \infty} A_{p, N}=1, \lim _{p \rightarrow \infty} \frac{\alpha}{\sqrt{p}}=\sqrt{\frac{N-2}{2}}, \lim _{p \rightarrow \infty} \frac{m}{\sqrt{p}}=\frac{1}{\sqrt{2(N-2)}}, \\
& \lim _{p \rightarrow \infty} D_{p}=\frac{1}{4(N-1)} .
\end{aligned}
$$

If precise constants are not necessary, we use the notation $A \approx p^{b}$ for some real number $b$ if there exist two positive constants $c_{1}$ and $c_{2}$ such that, $c_{1} \leq \frac{A}{p^{b}} \leq c_{2}$, for $p$ large. We also use the notation $A_{p}=O\left(p^{-b}\right)$ if there exists a constant $C$ not depending on $p$, such that $\left|A_{p}\right| \leq C p^{-b}$ for any large $p$. First, we provide an upper bound (for $p$ large) for the first intersection of the singular solution with the value 1 . Let us prove an auxiliary lemma first. 
Lemma 2.1 There exists $0<\tilde{c}<1$ such that for any sufficiently large $p$,

$$
P_{N}< \begin{cases}\frac{1}{2} \frac{1-e^{-\frac{(\alpha+8 m) \pi}{2 \beta}}}{1+e^{-\frac{(\alpha+8 m) \pi}{2 \beta}}} & \text { if } N<10, \\ \frac{1}{2} & \text { if } N \geq 10,\end{cases}
$$

where

$$
P_{N}:=\frac{\left|\phi\left(f\left(\tilde{\zeta}_{p}\right)\right)\right|}{f\left(\tilde{\zeta}_{p}\right)} \times \begin{cases}\frac{4}{(\alpha+8 m)^{2}+4 \beta^{2}}\left(1+e^{-\frac{(\alpha+8 m) \pi}{2 \beta}}\right) & \text { if } N<10, \\ \frac{1}{2 \beta\left(\frac{\alpha}{2}+4 m-\beta\right)} & \text { if } N \geq 10\end{cases}
$$

and

$$
r_{p}=e^{-m_{p} \zeta_{p}}, \quad \frac{\tilde{c}}{\sqrt{p}}=e^{-m_{p} \tilde{\zeta}_{p}}=\sqrt{\frac{f\left(\tilde{\zeta}_{p}\right)}{D_{p}}} .
$$

Proof First, notice that

$$
(\alpha+8 m)^{2}+4 \beta^{2} \approx p \text { for } N<10, \quad 2 \beta\left(\frac{\alpha}{2}+4 m-\beta\right) \approx p \text { for } N \geq 10,
$$

and

$$
\frac{\alpha+8 m}{2 \beta} \approx 1 \text { for } N<10 .
$$

In addition, since $f\left(\tilde{\zeta}_{p}\right)=D_{p} \tilde{c}^{2} / p$ and $D_{p} \approx 1$, we can choose $\tilde{c}$ sufficiently small such that $k:=D_{p} \tilde{c}^{2} \leq 1$. Then using that $p \mapsto(1+k / p)^{p}$ increases to $e^{k}$, we obtain

$$
\left|\phi\left(\frac{k}{p}\right)\right|=\left|\left(1+\frac{k}{p}\right)^{p}-k-1\right| \leq\left|e^{k}-k-1\right| \leq c_{N} k^{2} .
$$

Consequently,

$$
\frac{\left|\phi\left(f\left(\tilde{\zeta}_{p}\right)\right)\right|}{f\left(\tilde{\zeta}_{p}\right)}=\frac{p}{\tilde{c}^{2} D_{p}}\left|\phi\left(D_{p} \tilde{c}^{2} / p\right)\right| \leq c_{N} p D_{p} \tilde{c}^{2}
$$

and from (2.10) follows

$$
P_{N} \leq p D_{p} \tilde{c}^{2} \frac{C_{N}}{p}=C_{N} D_{p} \tilde{c}^{2}
$$

Hence, (2.7) is satisfied for some sufficiently small $\tilde{c}$ independent of $p$ as desired.

In the rest of the proof, we fix $\tilde{c}$ such that Lemma 2.1 holds. Fix any $\varepsilon_{0}>0$ and set

$$
\zeta_{1}^{*}:=\inf \left\{\zeta \geq \tilde{\zeta}_{p}:|\tilde{\eta}(z)| \leq\left(1+\varepsilon_{0}\right) P_{N} f(z) \text { for any } z \geq \zeta\right\}
$$

To simplify notation, we set $P_{N, \varepsilon_{0}}:=P_{N}\left(1+\varepsilon_{0}\right)$. First, we show that $\zeta_{1}^{*}$ is well-defined.

Lemma 2.2 For any $p>2^{*}-1$ and any $\varepsilon_{0}>0$, we have $\zeta_{1}^{*}<\infty$.

Proof Fix any $\varepsilon>0$. First, notice that

$$
\left\|G_{N}\right\|_{L^{1}} \leq C_{N, p}=\left\{\begin{array}{l}
\frac{2}{\alpha \beta}, \quad \text { if } N<10, \\
\frac{2}{\alpha^{2}} \frac{1}{\beta(\alpha-2 \beta)}, \quad \text { if } N>10,
\end{array}\right.
$$


and $C_{N, p} \approx p^{-1}$ if $N \neq 10$ and $C_{N, p} \approx p^{-1 / 2}$ if $N=10$. Using the representation formula (2.4) and Young's inequality for convolutions, we obtain

$$
\int_{\zeta}^{\infty}|\tilde{\eta}(\sigma)| d \sigma \leq C_{N, p} \int_{\zeta}^{\infty}|\tilde{g}(\sigma)| d \sigma
$$

Since the function $x \mapsto|\phi(x)| / x$ is increasing, then (2.11) implies

$$
\frac{|\phi(\eta)|}{\eta} \leq \frac{|\phi(\varepsilon / p)|}{\varepsilon / p} \leq c_{N} p \varepsilon \quad \text { for all } 0<\eta \leq \frac{\varepsilon}{p} .
$$

On the other hand, since $\eta(\zeta) \rightarrow 0$ as $\zeta \rightarrow \infty$ (see (2.2)), we deduce that there exists $\zeta_{0}>0$ depending on $p$ and $\eta$ such that $|\eta(\zeta)| \leq \varepsilon / p$ for any $\zeta \geq \zeta_{0}$, and consequently by the definition of $\tilde{\eta}$

$$
\mid \phi\left(\eta(\zeta)\left|\leq c_{N} p \varepsilon\right| \eta(\zeta) \mid \leq c_{N} p \varepsilon\left(|\tilde{\eta}(\zeta)|+\left|D_{p} e^{-2 m \sigma}\right|\right)\right.
$$

Recalling the definition of $\tilde{g}$ (see (2.3)), one has for $\sigma \geq \zeta_{0}$,

$$
|\tilde{g}(\sigma)| \leq\left(c_{N} \varepsilon p+m^{2} e^{-2 m \sigma}\right)\left(|\tilde{\eta}(\sigma)|+D_{p} e^{-2 m \sigma}\right) .
$$

Since $m \approx \sqrt{p}$ and $D_{p} e^{-2 m \zeta}=f(\zeta) \leq \varepsilon / p$ for $\zeta \geq \zeta_{0}$, then $m^{2} e^{-2 m \sigma} \leq c_{N} \varepsilon$, and therefore

$$
|\tilde{g}(\sigma)| \leq 2 c_{N} \varepsilon p\left(|\tilde{\eta}(\sigma)|+D_{p} e^{-2 m \sigma}\right) .
$$

Substituting this estimate into (2.13), we obtain, for $\zeta \geq \zeta_{0}$ and any sufficiently large $p \geq c_{0}$,

$$
\left(1-2 c_{N} \varepsilon p C_{N, p}\right) \int_{\xi}^{\infty}|\tilde{\eta}(\sigma)| d \sigma \leq \frac{c_{N} \varepsilon p D_{p}}{m} C_{N, p} e^{-2 m \zeta} .
$$

We decrease $\varepsilon_{0}$ if necessary to have

$$
\varepsilon_{0}<\frac{1}{4 c_{N} p C_{N, p}}, \quad \text { and therefore }\left(1-2 c_{N} \varepsilon p C_{N, p}\right) \geq \frac{1}{2}, \quad \text { for any } \varepsilon \in\left(0, \varepsilon_{0}\right) .
$$

Hence,

$$
\int_{\xi}^{\infty}|\tilde{\eta}(\sigma)| d \sigma \leq \frac{c_{N} D_{p}}{m} e^{-2 m \zeta} .
$$

Next, we use $\left\|G_{N}\right\|_{L^{\infty}} \leq C_{N}$ combined with (2.4), (2.14), (2.15) and Young convolution inequality to get that

$$
\begin{aligned}
& |\eta(\zeta)| \leq C_{N} \int_{\zeta}^{\infty}|\tilde{g}(\sigma)| d \sigma \leq C_{N} \varepsilon p \int_{\zeta}^{\infty}\left(D_{p} e^{-2 m \sigma}+|\tilde{\eta}(\sigma)|\right) d \sigma \\
& \quad \leq C_{N} \varepsilon p\left(\frac{D_{p}}{m}+\frac{c_{N} D_{p}}{m}\right) e^{-2 m \zeta} .
\end{aligned}
$$

Then, the definition of $\varepsilon$ yields

$$
|\eta(\zeta)| \leq \frac{c_{N} D_{p}}{m C_{N, p}} e^{-2 m \zeta} \quad \text { for any } \zeta \geq \zeta_{0} .
$$

Since $D_{p} \approx 1, m \approx \sqrt{p}$, and $C_{N, p} \geq 1$ for large $p$, we obtain the desired conclusion.

Lemma 2.3 For any small $\varepsilon_{0}>0$, there exists $p_{0}>0$ such that, for each $p \geq p_{0}$, we have $\eta \leq 0$ on $\left[\zeta_{1}^{*}, \infty\right)$ where $\zeta_{1}^{*}$ is defined in $(2.12)$. 
Proof We first assume that $N \leq 10$. We rewrite (2.4) as

$$
\tilde{\eta}(\zeta)=\int_{\zeta}^{\infty} G_{N}(\sigma-\zeta) \tilde{g}(\sigma) d \sigma=: \int_{\zeta}^{\infty} F(\zeta, \sigma) d \sigma
$$

and

$$
\begin{aligned}
\int_{\zeta}^{\infty} F(\zeta, \sigma) d \sigma & =\sum_{k=0}^{\infty} \int_{\zeta+\frac{2 k \pi}{\beta}}^{\zeta+\frac{(2 k+1) \pi}{\beta}} F(\zeta, \sigma) d \sigma+\int_{\zeta+\frac{(2 k+1) \pi}{\beta}}^{\zeta+\frac{(2 k+2) \pi}{\beta}} F(\zeta, \sigma) d \sigma \\
& =\sum_{k=0}^{\infty} \int_{\zeta+\frac{2 k \pi}{\beta}}^{\zeta+\frac{(2 k+1) \pi}{\beta}} F(\zeta, \sigma)+F\left(\zeta, \sigma+\frac{\pi}{\beta}\right) d \sigma
\end{aligned}
$$

where

$$
F(\zeta, \sigma)+F\left(\zeta, \sigma+\frac{\pi}{\beta}\right)=G_{N}(\sigma-\zeta)\left(\tilde{g}(\sigma)-e^{-\frac{\alpha \pi}{2 \beta}} \tilde{g}\left(\sigma+\frac{\pi}{\beta}\right)\right)
$$

Recall, for any $\sigma \geq \zeta_{1}^{*}$ we have $|\tilde{\eta}(\sigma)| \leq P_{N, \varepsilon_{0}} f(\sigma)$, with $1>P_{N, \varepsilon_{0}}$ for any sufficiently small $\varepsilon_{0}>0$. Since $\phi$ is decreasing on $(0, \infty)$ and $f \pm \tilde{\eta} \geq 0$ on $\left[\zeta_{1}^{*}, \infty\right)$, one has

$$
\begin{aligned}
& \phi((f+\tilde{\eta})(\sigma))-e^{-\frac{\alpha \pi}{2 \beta}} \phi\left((f+\tilde{\eta})\left(\sigma+\frac{\pi}{\beta}\right)\right) \\
& \leq \phi((f-|\tilde{\eta}|)(\sigma))-e^{-\frac{\alpha \pi}{2 \beta}} \phi\left((f+|\tilde{\eta}|)\left(\sigma+\frac{\pi}{\beta}\right)\right) \\
& \leq \phi\left(\left(1-P_{N, \varepsilon_{0}}\right) f(\sigma)\right)-e^{-\frac{\alpha \pi}{2 \beta}} \phi\left(\left(1+P_{N, \varepsilon_{0}}\right) f\left(\sigma+\frac{\pi}{\beta}\right)\right) \\
& \leq \phi\left(\left(1-P_{N, \varepsilon_{0}}\right) f(\sigma)\right)-e^{-\frac{\alpha \pi}{2 \beta}} \phi\left(\left(1+P_{N, \varepsilon_{0}}\right) e^{-\frac{2 \pi m}{\beta}} f(\sigma)\right) .
\end{aligned}
$$

We claim that for any sufficiently small $\varepsilon_{0}, \varepsilon_{1}>0$ and any sufficiently large $m$ (that is large $p$ ), one has

$$
\begin{aligned}
& \phi\left(\left(1-P_{N, \varepsilon_{0}}\right) z\right) \leq e^{-\frac{\alpha \pi}{2 \beta}} \phi\left(\left(1+P_{N, \varepsilon_{0}}\right) e^{-\frac{2 \pi m}{\beta}} z\right)-2\left(1+P_{N, \varepsilon_{0}}\right) \frac{m^{2}}{D_{p}} z^{2}, \\
& \text { for any } z \in\left[0, K_{M} / p\right] .
\end{aligned}
$$

Indeed, it is easy to check that both value and the value of the derivatives of both sides in (2.18) vanish at $z=0$. Thus, it suffices to verify that the second derivative of the right hand side is larger than the second derivative of the left hand side on the interval $\left[0, K_{M} / p\right]$. It is equivalent to

$$
\begin{aligned}
& p(p-1)\left(1-P_{N, \varepsilon_{0}}\right)^{2}\left(1+\left(1-P_{N, \varepsilon_{0}}\right) z\right)^{p-2} \\
& \geq p(p-1)\left(1+P_{N, \varepsilon_{0}}\right)^{2} e^{-\frac{\pi}{2 \beta}(\alpha+8 m)}\left(1+\left(1+P_{N, \varepsilon_{0}}\right) e^{-\frac{2 \pi m}{\beta}} z\right)^{p-2}+4\left(1+P_{N, \varepsilon_{0}}\right) \frac{m^{2}}{D_{p}} .
\end{aligned}
$$

However, by (2.7),

$$
P_{N}<\frac{1}{2} \frac{1-e^{-\frac{\pi}{2 \beta}(\alpha+8 m)}}{1+e^{-\frac{\pi}{2 \beta}(\alpha+8 m)}}<\frac{1}{2}
$$


and by (2.6)

$$
\begin{aligned}
\frac{\alpha+8 m}{2 \beta} & =\frac{N+6}{\sqrt{(N-2)(10-N)}}+O(1) \geq 2 \sqrt{2}+O(1) \quad \text { for } N \in[3,10), \\
\frac{m}{\beta} & =\frac{2}{\sqrt{(N-2)(10-N)}}+O(1) \geq \frac{1}{2}+O(1) \quad \text { for } N \in[3,10) .
\end{aligned}
$$

For $N=10$, the left hand side diverges to infinity, so the latter estimates are still valid. Thus, for any sufficiently small $\varepsilon_{0}$ and large $p$, we have

$$
\begin{aligned}
1-P_{N, \varepsilon_{0}} & \geq \frac{1}{2} \geq \frac{3}{2} e^{-\pi+O(1)} \geq\left(1+P_{N, \varepsilon_{0}}\right) e^{-\frac{2 \pi m}{\beta}} \\
\left(1-P_{N, \varepsilon_{0}}\right)^{2} & \geq \frac{1}{4} \geq \frac{9}{2} e^{-2 \sqrt{2} \pi+O(1)} \geq 2\left(1+P_{N, \varepsilon_{0}}\right)^{2} e^{-\frac{\pi}{2 \beta}(\alpha+8 m)} .
\end{aligned}
$$

So, we obtain, for any small $\varepsilon_{0}>0$, and large $p$

$$
\begin{aligned}
(1- & \left.P_{N, \varepsilon_{0}}\right)^{2}\left(1+\left(1-P_{N, \varepsilon_{0}}\right) z\right)^{p-2} \\
& -\left(1+P_{N, \varepsilon_{0}}\right)^{2} e^{-\frac{\pi}{2 \beta}(\alpha+8 m)}\left(1+\left(1+P_{N, \varepsilon_{0}}\right) e^{-\frac{2 \pi m}{\beta}} z\right)^{p-2} \\
\geq & \frac{1}{2}\left(1-P_{N, \varepsilon_{0}}\right)^{2}\left(1+\left(1-P_{N, \varepsilon_{0}}\right) z\right)^{p-2} \geq \frac{1}{2}\left(1-P_{N, \varepsilon_{0}}\right)^{2} .
\end{aligned}
$$

Since $m^{2} \approx p,(2.18)$ follows for any sufficiently large $p$.

In addition, using that $f$ is decreasing and that $|\tilde{\eta}(\sigma)| \leq P_{N, \varepsilon_{0}} f(\sigma)$, we have, for $\sigma \geq \zeta_{1}^{*}$,

$$
\begin{aligned}
m^{2} e^{-2 m \sigma}\left((\tilde{\eta}+f)(\sigma)-e^{-\frac{\pi}{\beta}(2 m+\alpha / 2)}(\tilde{\eta}+f)\left(\sigma+\frac{\pi}{\beta}\right)\right) & \leq 2\left(1+P_{N, \varepsilon_{0}}\right) m^{2} e^{-2 m \sigma} f(\sigma) \\
& =2\left(1+P_{N, \varepsilon_{0}}\right) \frac{m^{2}}{D_{p}} f^{2}(\sigma)
\end{aligned}
$$

Therefore, recalling that $\tilde{g}(\zeta)=\phi(\eta(\zeta))+m^{2} e^{-2 m \zeta} \eta(\zeta)$, the previous bound combined with (2.18) implies

$$
\tilde{g}(\sigma)-e^{-\frac{\alpha \pi}{2 \beta}} \tilde{g}\left(\sigma+\frac{\pi}{\beta}\right) \leq 0
$$

Since $G_{N} \geq 0$ on $\left(\zeta+\frac{2 k \pi}{\beta}, \zeta+\frac{2(k+1) \pi}{\beta}\right)$, we obtain that

$$
F(\zeta, \sigma)+F\left(\zeta, \sigma+\frac{\pi}{\beta}\right) \leq 0 .
$$

Using (2.17) and (2.16), this established the proof for $N \leq 10$.

Next, assume $N>10$ and notice that $G_{N} \geq 0$ in this case. Also, since $|\tilde{\eta}(\sigma)| \leq P_{N, \varepsilon_{0}} f(\sigma)$ on $\left[\zeta_{1}^{*}, \infty\right)$ and $P_{N, \varepsilon_{0}}<1$ for any sufficiently small $\varepsilon_{0}$, we obtain that $\eta=f+\tilde{\eta} \geq 0$ on $\left[\zeta_{1}^{*}, \infty\right)$. Since $(1+x)^{p}-1-p x \geq \frac{p(p-1)}{2} x^{2}$ for $x \geq 0$, then for any $\zeta \geq \zeta_{1}^{*}$

$$
\tilde{\eta}(\zeta) \leq \int_{\zeta}^{\infty} G_{N}(\sigma-\zeta)\left(m^{2} e^{-2 \sigma} \eta(\sigma)-\frac{p(p-1)}{2} \eta^{2}(\sigma)\right) d \sigma .
$$


Also, since $\eta \geq 0$, we have

$$
\begin{aligned}
m^{2} e^{-2 \sigma} \eta(\sigma)-\frac{p(p-1)}{2} \eta^{2}(\sigma) & \leq \eta(\sigma)\left(m^{2} \frac{f(\sigma)}{D_{p}}-\frac{p(p-1)}{2}(f(\sigma)-|\tilde{\eta}(\sigma)|)\right) \\
& \leq \eta(\sigma) f(\sigma)\left(\frac{m^{2}}{D_{p}}-\frac{p(p-1)}{2}\left(1-P_{N, \varepsilon_{0}}\right)\right) \leq 0
\end{aligned}
$$

where we used $\frac{m^{2}}{D_{p}} \approx p$ in the last inequality. Thus $\tilde{\eta}(\zeta) \leq 0$ for each $\zeta \geq \zeta_{1}^{*}$ as desired.

Lemma 2.4 For any sufficiently small $\varepsilon_{0}>0$, there exists $p_{0}$ such that, for each $p \geq p_{0}$, we have $\zeta_{1}^{*}=\tilde{\zeta}_{p}$, where $\zeta_{1}^{*}$ is defined in (2.12). In particular, $\left|\tilde{\eta}\left(\tilde{\zeta}_{p}\right)\right|<\frac{f\left(\tilde{\zeta}_{p}\right)}{2}$.

Proof In Lemma 2.3, we proved that $\tilde{\eta} \leq 0$ on $\left(\zeta_{1}^{*}, \infty\right)$. In order to obtain an estimate on $|\tilde{\eta}|$, we need a lower bound on $\tilde{\eta}$.

First, let us assume that $N \leq 10$. Since $G_{N}(\sigma-\zeta) \leq 0$ on the interval $\left(\zeta+\frac{(2 k+1) \pi}{\beta}, \zeta+\frac{(2 k+2) \pi}{\beta}\right),(2.19)$ and (2.17) yield on such interval

$$
F(\zeta, \sigma)+F\left(\zeta, \sigma+\frac{\pi}{\beta}\right) \geq 0 .
$$

Consequently, by using that $\phi$ is decreasing and $\tilde{\eta} \leq 0$, we obtain, for any $\zeta \geq \zeta_{1}^{*}$,

$$
\begin{aligned}
\tilde{\eta}(\zeta) & \geq \int_{\zeta}^{\zeta+\frac{\pi}{\beta}} G_{N}(\sigma-\zeta) \phi((f+\tilde{\eta})(\sigma)) d \sigma+m^{2} \int_{\zeta}^{\infty} G_{N}(\sigma-\zeta) e^{-2 m \sigma}(\tilde{\eta}+f)(\sigma) d \sigma \\
& \geq \int_{\zeta}^{\zeta+\frac{\pi}{\beta}} G_{N}(\sigma-\zeta) \phi(f(\sigma)) d \sigma-m^{2}\left(1+P_{N, \varepsilon_{0}}\right) \int_{\zeta}^{\infty}\left|G_{N}(\sigma-\zeta)\right| e^{-2 m \sigma} f(\sigma) d \sigma .
\end{aligned}
$$

Using the explicit forms of $G_{N}$ and $f$, a direct computation allows us to estimate the second term

$$
m^{2}\left(1+P_{N, \varepsilon_{0}}\right) \int_{\zeta}^{\infty}\left|G_{N}(\sigma-\zeta)\right| e^{-2 m \sigma} f(\sigma) d \sigma \leq\left(1+P_{N, \varepsilon_{0}}\right) m^{2} \frac{D_{p}}{(4 m+\alpha / 2) \beta} e^{-4 m \zeta} .
$$

In order to estimate the first term on the right hand side of (2.20), we use that $x \mapsto \phi(x) / x^{2}$ is decreasing, and therefore for any $y \geq x>0$,

$$
\frac{\phi(x)}{x^{2}} \geq \frac{\phi(y)}{y^{2}}
$$

which implies

$$
\phi(f(\sigma)) \geq \phi(f(\zeta))\left(\frac{f(\sigma)}{f(\zeta)}\right)^{2}=\phi(f(\zeta)) e^{-4 m(\sigma-\zeta)} .
$$

Thus, inserting the two previous estimates into (2.20), we obtain for any $\zeta \geq \zeta_{1}^{*}$

$$
\begin{aligned}
\tilde{\eta}(\zeta) \geq & \frac{\phi(f(\zeta))}{\beta} \int_{\zeta}^{\zeta+\frac{\pi}{\beta}} e^{-\left(\frac{\alpha}{2}+4 m\right)(\sigma-\zeta)} \sin (\beta(\sigma-\zeta)) d \sigma \\
& -\left(1+P_{N, \varepsilon_{0}}\right) m^{2} \frac{D_{p}}{(4 m+\alpha / 2) \beta} e^{-4 m \zeta} \\
= & \frac{4 \phi(f(\zeta))}{(\alpha+8 m)^{2}+4 \beta^{2}}\left(1+e^{-\frac{(\alpha+8 m) \pi}{2 \beta}}\right)-\left(1+P_{N, \varepsilon_{0}}\right) m^{2} \frac{D_{p}}{(4 m+\alpha / 2) \beta} e^{-4 m \zeta}
\end{aligned}
$$


Since $\zeta_{1}^{*} \geq \tilde{\zeta}_{p}$, we have $f(\zeta) \leq C / p$, for any $\zeta \geq \zeta_{1}^{*}$. Thus, there exists a constant $C_{N}>0$, not depending on $p$, such that

$$
\left(1+P_{N, \varepsilon_{0}}\right) m^{2} \frac{D_{p}}{(4 m+\alpha / 2) \beta} e^{-4 m \zeta} \leq C_{N} p^{1 / 2} \beta^{-1} f^{2}(\zeta) \leq C_{N} p^{-1 / 2} f(\zeta) .
$$

Using again that $\tilde{\eta} \leq 0$ and $x \mapsto \phi(x) / x$ is decreasing and the definition of $P_{N}$ (see (2.8)), we obtain, for any $\zeta \geq \tilde{\zeta}_{p}$ and sufficiently large $p$,

$$
\begin{aligned}
& |\tilde{\eta}(\zeta)| \leq \frac{4|\phi(f(\zeta))|}{\left((\alpha+8)^{2}+4 \beta^{2}\right) f(\zeta)}\left(1+e^{-\frac{(\alpha+8) \pi}{2 \beta}}\right) f(\zeta)+\frac{C}{p^{\frac{1}{2}}} f(\zeta) \\
& \quad \leq\left(P_{N}+\frac{C}{p^{\frac{1}{2}}}\right) f(\zeta)<\left(1+\frac{\varepsilon_{0}}{2}\right) P_{N} f(\zeta) .
\end{aligned}
$$

If $\zeta_{1}^{*}>\tilde{\zeta}_{p}$, then, by continuity and (2.21), $|\tilde{\eta}(\zeta)| \leq\left(1+\varepsilon_{0}\right) P_{N}|f(\zeta)|$ holds for any $\tilde{\zeta}_{p} \leq \zeta \leq \zeta_{1}^{*}$ sufficiently close to $\zeta_{1}^{*}$, a contradiction to the definition of $\zeta_{1}^{*}$. Thus $\zeta_{1}^{*}=\tilde{\zeta}_{p}$ as desired.

If $N>10$, using $G_{N} \geq 0$, the monotonicity of $\phi$, and $\tilde{\eta} \leq 0$ as above, we obtain, for any $\zeta \geq \zeta_{1}^{*}$

$$
\begin{aligned}
\tilde{\eta}(\zeta) & \geq \int_{\zeta}^{\infty} G_{N}(\sigma-\zeta)\left(\phi(f(\zeta)) e^{-4 m(\sigma-\zeta)}-m^{2}\left(1+P_{N, \varepsilon_{0}}\right) e^{-2 m \sigma} f(\sigma)\right) d \sigma \\
& \geq \phi(f(\zeta)) \int_{\zeta}^{\infty} G_{N}(\sigma-\zeta) e^{-4 m(\sigma-\zeta)} d \sigma-\frac{1}{2 \beta\left|\frac{\alpha}{2}-\beta+4 m\right|} f^{2}(\zeta),
\end{aligned}
$$

Then, one has

$$
\tilde{\eta}(\zeta) \geq \frac{1}{2 \beta\left(\frac{\alpha}{2}+4 m-\beta\right)} \phi(f(\zeta))-O\left(p^{-1}\right) f^{2}(\zeta)
$$

Proceeding as above, we find

$$
|\tilde{\eta}(\zeta)| \leq \frac{\phi(f(\zeta))}{2 \beta\left(\frac{\alpha}{2}+4 m-\beta\right) f(\zeta)} f(\zeta)+\frac{C}{p} f^{2}(\zeta)<\left(1+\frac{\varepsilon_{0}}{2}\right) P_{N} f(\zeta) .
$$

And the proof is concluded as in the previous case.

Remark 2.5 In the Lemma 2.4, we proved that

$$
0 \geq \tilde{\eta}(\zeta) \geq-\left(1+\varepsilon_{0}\right) P_{N} f(\zeta) \quad \text { for any } \zeta \geq \tilde{\zeta}_{p}
$$

which combined with $P_{N} \leq \frac{1}{2}$ imply

$$
0 \leq \eta \leq f(\zeta) \quad \text { for any } \zeta \geq \tilde{\zeta}_{p}
$$

In the original variables, we have

$$
A_{p, N} r^{-\theta} \leq U_{p}^{*}(r) \leq A_{p, N} r^{-\theta}\left(1+D_{p} r^{2}\right) \quad \text { for any } r \leq \tilde{c} / \sqrt{p} .
$$

The importance of this bound is in the estimate on $U_{p}^{*}$ on an explicit interval.

Proposition 2.6 For any fixed $i \in \mathbb{N}$, we have

$$
R_{p}^{i} \rightarrow 0 \text {, as } p \rightarrow \infty .
$$


Proof Assume $\tilde{c}$ is as in Lemma 2.1 and denote $\tilde{r}_{p}=\tilde{c} / \sqrt{p}$ and $\tilde{\zeta}_{p}=-m^{-1} \ln \tilde{r}_{p}$. Then, Remark 2.5 holds on the interval $\left(0, \tilde{r}_{p}\right]$. As above, we denote by $C_{N}$ constants depending on $N$ but not on $p$.

First assume $N \leq 10$. Observe that (2.11), implies $|\phi(z)| \leq C_{N} p^{2} z^{2}$ for $z \leq c_{N} / p$ and consequently Lemma 2.4 yields $|\tilde{g}(\zeta)| \leq C_{n} p^{2} e^{-4 m \zeta}$ for any $\zeta \geq \tilde{\zeta}_{p}$. Then, taking the derivative of the representation formula (2.4), using that $\tilde{\eta} \leq 0$, asymptotics (2.6), and the definition of $\tilde{\zeta}_{p}$ (see (2.9)), we have

$$
\begin{aligned}
\tilde{\eta}^{\prime}\left(\tilde{\zeta}_{p}\right) & =\frac{\alpha}{2} \tilde{\eta}\left(\tilde{\zeta}_{p}\right)-e^{(\alpha / 2) \tilde{\zeta}_{p}} \int_{\tilde{\zeta}_{p}}^{\infty} e^{-(\alpha / 2) \sigma} \cos \left(\beta\left(\sigma-\tilde{\zeta}_{p}\right)\right) \tilde{g}(\sigma) d \sigma \\
& \leq C_{N} p^{2} e^{(\alpha / 2) \tilde{\zeta}_{p}} \int_{\tilde{\zeta}_{p}}^{\infty} e^{-(\alpha / 2) \sigma} e^{-4 m \sigma} d \sigma \\
& \leq C_{N} p^{3 / 2} e^{-4 m \tilde{\zeta}_{p}} \leq \frac{C_{N}}{\sqrt{p}}
\end{aligned}
$$

The same estimate holds true for $N>10$ since

$$
\begin{aligned}
\int_{\tilde{\zeta}_{p}}^{\infty} e^{-(\alpha / 2) \sigma} \cosh \left(\beta\left(\sigma-\tilde{\zeta}_{p}\right) e^{-4 m \sigma} d \sigma\right. & \leq \int_{\tilde{\zeta}_{p}}^{\infty} e^{-(\alpha / 2) \sigma} e^{\beta\left(\sigma-\tilde{\zeta}_{p}\right)} e^{-4 m \sigma} d \sigma \\
& \leq C_{N} p^{-1 / 2} e^{-(\alpha / 2) \tilde{\zeta}_{p}} e^{-4 m \tilde{\zeta}_{p}} .
\end{aligned}
$$

Thus, we have, using that $U_{p}^{*}(r)=A_{p, N} r^{-\theta}\left(\tilde{\eta}(\zeta)+1+D_{p} e^{-2 m \zeta}\right)$, (2.6), Lemma 2.4, (2.22), and the definition of $\tilde{r}_{p}$

$$
\begin{aligned}
\left|\left(U_{p}^{*}\right)^{\prime}\left(\tilde{r}_{p}\right)\right| & =A_{p, N} \tilde{r}_{p}^{-\theta}\left|(-\theta)\left(\frac{\tilde{\eta}\left(\tilde{\zeta}_{p}\right)+1}{\tilde{r}_{p}}+D_{p} \tilde{r}_{p}\right)-\frac{\tilde{\eta}^{\prime}\left(\tilde{\zeta}_{p}\right)}{m \tilde{r}_{p}}+2 D_{p} \tilde{r}_{p}\right| \\
& \leq C_{N}\left(\tilde{r}_{p}+\left(p \tilde{r}_{p}\right)^{-1}\left(1+\left|\tilde{\eta}\left(\tilde{\zeta}_{p}\right)\right|\right)+\left|\tilde{\eta}^{\prime}\left(\tilde{\zeta}_{p}\right)\right| \tilde{r}_{p}^{-1} p^{-1 / 2}\right) \\
& \leq C_{N} p^{-1 / 2} \rightarrow 0, \text { as } p \rightarrow \infty .
\end{aligned}
$$

In addition, Remark 2.5 implies

$$
A_{p, N}\left(\frac{p}{\tilde{c}^{2}}\right)^{\frac{1}{p-1}} \leq U_{p}^{*}\left(\tilde{r}_{p}\right) \leq A_{p, N}\left(\frac{p}{\tilde{c}^{2}}\right)^{\frac{1}{p-1}}\left(1+D_{p} \frac{\tilde{c}^{2}}{p}\right),
$$

and consequently $U_{p}^{*}\left(\tilde{r}_{p}\right) \rightarrow 1$ as $p \rightarrow \infty$. Also, we have

$$
A_{p, N}^{p+1}\left(\frac{p}{\tilde{c}^{2}}\right)^{\frac{p+1}{p-1}} \leq\left(U_{p}^{*}\left(\tilde{r}_{p}\right)\right)^{p+1} \leq A_{p, N}^{p+1}\left(\frac{p}{\tilde{c}^{2}}\right)^{\frac{p+1}{p-1}}\left(1+D_{p} \frac{\tilde{c}^{2}}{p}\right)^{p+1} .
$$

Since $A_{p, N}^{p+1} \approx(p-1)^{-\frac{p+1}{p-1}}$, we obtain that $\left(U_{p}^{*}\left(\tilde{r}_{p}\right)\right)^{p+1} \approx 1$, and therefore $\left(U_{p}^{*}\left(\tilde{r}_{p}\right)\right)^{p+1} /(p+$ $1) \approx p^{-1}$ as $p \rightarrow \infty$.

Next, we will prove more precise estimate. Since the function

$$
r \mapsto E(r)=\frac{\left(\left(U_{p}^{*}\right)^{\prime}(r)\right)^{2}}{2}-\frac{1}{2}\left(U_{p}^{*}\right)^{2}(r)+\frac{\left(U_{p}^{*}\right)^{p+1}(r)}{p+1}
$$

is non-increasing, then by the above estimates, one has, for any $r \geq \tilde{r}_{p}$,

$$
\left(\left(U_{p}^{*}\right)^{\prime}\left(\tilde{r}_{p}\right)\right)^{2}-\frac{1}{2}+\mu_{p} \geq E\left(\tilde{r}_{p}\right) \geq E(r) \geq-\frac{\left(U_{p}^{*}\right)^{2}(r)}{2},
$$


where $\mu_{p} \rightarrow 0$ as $p \rightarrow \infty$. Hence, from (2.23) follows

$$
1-\left(U_{p}^{*}\right)^{2}(r) \leq 2 \mu_{p},
$$

and therefore $\left(1-\left(U_{p}^{*}\right)^{2}(r)\right)_{+} \rightarrow 0$ as $p \rightarrow \infty$, where $h_{+}=\max \{h, 0\}$ denotes the positive part of a function $h$. On the other hand, if there is $\varepsilon^{*}>0$ and $r_{p}^{*}>\tilde{r}_{p}$ such that $U_{p}^{*}\left(r_{p}^{*}\right) \geq 1+\varepsilon^{*}$, then

$$
E\left(r_{p}^{*}\right) \geq-\frac{1}{2}\left(U_{p}^{*}\right)^{2}(r)+\frac{\left(U_{p}^{*}\right)^{p+1}(r)}{p+1} \rightarrow \infty \quad \text { as } p \rightarrow \infty,
$$

a contradiction to $E\left(r_{p}^{*}\right) \leq E\left(\tilde{r}_{p}\right) \leq C_{N}$.

Overall we proved that $\left|U_{p}^{*}(r)-1\right| \rightarrow 0$, for all $r \geq \tilde{r}_{p}$. Recall that $w(r)=r^{\frac{N-1}{2}}\left(U_{p}^{*}(r)-\right.$ 1) (see (2.5)) satisfies

$$
w^{\prime \prime}+\left(\frac{\left(U_{p}^{*}\right)^{p}-U_{p}^{*}}{U_{p}^{*}-1}-\frac{(N-1)(N-3)}{r^{2}}\right) w=0 .
$$

Fix $a>0$ and denote $I_{a}:=\left[\frac{a}{4}, a\right]$. Choose any $r \in I_{a}$. Since $\left|U_{p}^{*}(r)-1\right| \rightarrow 0$ locally uniformly, we have

$$
\frac{\left(U_{p}^{*}(r)\right)^{p}-U_{p}^{*}(r)}{U_{p}^{*}(r)-1} \geq p / 2, \quad \text { on } I_{a} .
$$

Fix a large $A>0$ depending on $a$ as specified below. Then, for sufficiently large $p>2^{*}-1$ depending on $a$ and $A$, one has

$$
\frac{\left(U_{p}^{*}(r)\right)^{p}-U_{p}^{*}(r)}{U_{p}^{*}(r)-1}-\frac{(N-1)(N-3)}{4 r^{2}} \geq A-C_{N, a} \quad \text { for any } \quad r \in I_{a}:=\left[\frac{a}{4}, a\right] .
$$

Thus, given $a>0$ and an integer $i>0$, we choose $A$ large enough such that a solution of the equation $z^{\prime \prime}+\left(A-C_{N, a}\right) z=0$ has at least $i+2$ zeros on $I_{a}$. By Sturm-Picone oscillation theorem, the function $w$ has at least $i+1$ zeros on $I_{a}$. Consequently, $U_{p}^{*}(r)=1$ has at least $i+1$ solutions on $I_{a}$, and therefore $U_{p}^{*}$ has at least $i$ critical points on $I_{a}$. In a different notation, for any $j \in\{1, \ldots, i\}$ and any $a>0$, one has $R_{p}^{j}<a$, for any sufficiently large $p>2^{*}-1$.

Remark 2.7 By (2.23), we have

$$
\left|\left(U_{p}^{*}\right)^{\prime}(r)+\theta A_{p, N} r^{-1-\theta}\right| \leq\left|A_{p, N} r^{-\theta}\left((-\theta)\left(\frac{\tilde{\eta}(\zeta)}{r}+D_{p} r\right)-\frac{\tilde{\eta}^{\prime}(\zeta)}{m r}+2 D_{p} r\right)\right| .
$$

By Remark 2.5 and (2.22), one has $|\eta(\zeta)| \leq f(\zeta) \leq C_{N} r^{2}$ and $\left|\eta^{\prime}(\zeta)\right| \leq C_{N} \sqrt{p} r^{2}$ for any $r \leq \frac{\tilde{c}}{\sqrt{p}}$. Thus,

$$
\left|\left(U_{p}^{*}\right)^{\prime}(r)+\theta A_{p, N} r^{-1-\theta}\right| \leq C_{N} r^{1-\theta} \text {, for any } r \leq \frac{\tilde{c}}{\sqrt{p}} .
$$

Proposition 2.8 For any $i \in \mathbb{N}$, the function $p \rightarrow R_{p}^{i}$ is continuous. 
Proof Let $p^{*}>2^{*}-1$. Fix any open interval $I_{0}=(A, B)$ such that $0<A<B<\infty$ and without loss of generality assume that $A<\tilde{c} /(2 p)$. Then, by Remark 2.5 , there is $\delta>0$ such that, for any $p \in\left(p^{*}-\delta, p^{*}+\delta\right)$, one has

$$
\left|U_{p}^{*}(A)\right| \leq C_{N}
$$

If $r \leq R_{p}^{1}$, since $U_{p}^{*}$ is decreasing and positive (see [23, Theorem A.3]) on $\left(0, R_{p}^{1}\right)$, we have $\left|U_{p}^{*}(r)\right| \leq C_{N}$, for any $r \in\left(A, R_{p}^{1}\right)$. If $r>R_{p}^{1}$, we use the fact that the functional

$$
E(r)=\frac{\left(\left(U_{p}^{*}\right)^{\prime}(r)\right)^{2}}{2}-\frac{\left(U_{p}^{*}(r)\right)^{2}}{2}+\frac{\left(U_{p}^{*}(r)\right)^{p+1}}{p+1},
$$

is decreasing. Since $U_{p}^{*}\left(R_{p}^{1}\right) \leq 1$ by [23, Lemma 4.8], this implies for any $r \geq R_{p}^{1}$ that

$$
\frac{\left(U_{p}^{*}(r)\right)^{p+1}}{p+1}-\frac{\left(U_{p}^{*}(r)\right)^{2}}{2} \leq \frac{\left(U_{p}^{*}\left(R_{p}^{1}\right)\right)^{p+1}}{p+1} \leq \frac{1}{p+1} .
$$

Thus, also in this case, we have $\left|U_{p}^{*}\right| \leq C_{N}$ on $(A, B)$. Overall, we showed that

$$
\sup _{p \in\left(p^{*}-\delta, p^{*}+\delta\right)} \sup _{(A, B)} U_{p}^{*} \leq C(A) .
$$

Then, elliptic regularity theory implies that, for any $q>1$,

$$
\left\|U_{p}^{*}\right\|_{W^{3, q}\left(I_{0}\right)} \leq C\left(N, q, A, B-A, p^{*}, \delta\right), \quad \text { for any } p \in\left(p^{*}-\delta, p^{*}+\delta\right) .
$$

Let $\alpha_{0} \in(0,1)$. We choose $q_{0}>0$ large enough such that $W^{3, q_{0}}\left(I_{0}\right) \hookrightarrow C^{2+\alpha_{0}}\left(I_{0}\right)$. Let $\left(p_{n}\right)$ be a sequence such that $p_{n} \rightarrow p^{*}$ when $n \rightarrow \infty$. Thanks to (2.24), using Arzela-Ascoli Theorem, there exists a subsequence $\left(p_{n}\right)$ such that $U_{p_{n}}^{*} \rightarrow w$, as $n \rightarrow \infty$, in $C^{2}\left(I_{0}\right)$. From the uniform bound (2.24) follows

$$
\left|\left(U_{p_{n}}^{*}\right)^{p_{n}}(s)-w^{p^{*}}(s)\right| \leq\left|\left(U_{p_{n}}^{*}\right)^{p_{n}}(s)-\left(U_{p_{n}}^{*}\right)^{p^{*}}(s)\right|+\left|\left(U_{p_{n}}^{*}\right)^{p^{*}}(s)-w^{p^{*}}(s)\right|,
$$

we deduce that $w$ satisfies the equation

$$
-\Delta w+w=w^{p^{*}}, \quad \text { in } I_{0} .
$$

Since $I_{0}$ is an arbitrary compact interval, proceeding as above and using standard diagonal arguments, we obtain the existence of a subsequence $\left(p_{n}\right)_{n}, p_{n} \in\left(p^{*}-\delta, p^{*}+\delta\right)$, for all $n \in \mathbb{N}$, such that $U_{p_{n}}^{*} \rightarrow w$, as $n \rightarrow \infty$, in $C_{l o c}^{2}((0, \infty))$, for some function $w$ satisfying

$$
-\Delta w+w=w^{p^{*}} \quad \text { in }(0, \infty) .
$$

Next, we claim that $w$ is in fact equal to $U_{p^{*}}^{*}$. Using the uniqueness of solution to (1.6) (see Theorem 1.3), it is sufficient to show that

$$
\lim _{r \rightarrow 0^{+}} r^{\theta_{p^{*}}} w(r)=A_{p^{*}, N}
$$

where $\theta_{p^{*}}=\frac{2}{p-1}$. However if $p>2$, by Remark 2.5 for any $\varepsilon>0$, there is $r_{0}(\varepsilon)$ independent of $p \in\left(p^{*}-\delta, p^{*}+\delta\right)$ such that

$$
A_{p, N} r^{-\theta_{p}} \leq U_{p}^{*}(r) \leq A_{p, N} r^{-\theta_{p}}+\varepsilon, \quad \text { for all } \quad r \in\left(0, r_{0}(\varepsilon)\right) .
$$


Clearly $A_{p_{n}, N} \rightarrow A_{p^{*}, N}, \theta_{p_{n}} \rightarrow \theta_{p^{*}}$, when $n \rightarrow \infty$ and using that $U_{p_{n}}^{*} \rightarrow w$ in $C_{\text {loc }}^{2}\left(\left(0, r_{0}(\varepsilon)\right)\right)$, we obtain

$$
A_{p^{*}, N} r^{-\theta_{p^{*}}} \leq w(r) \leq A_{p^{*}, N} r^{-\theta_{p^{*}}}+\varepsilon, \text { for all } r \in\left(0, r_{0}(\varepsilon)\right) .
$$

Since $\varepsilon>0$ is arbitrary, we conclude that (2.25) holds, and therefore $w=U_{p^{*}}^{*}$ by uniqueness. Hence,

$$
U_{p}^{*} \rightarrow U_{p^{*}}^{*}, \quad \text { as } \quad p \rightarrow p^{*}, \quad \text { in } C_{l o c}^{2}((0, \infty)) .
$$

Finally, we prove the continuity of the function $p \rightarrow R_{p}^{i}$. In the following, we assume that $R_{p^{*}}^{i}$ is a local minimum of $U_{p^{*}}^{*}$, the case of local maximum follows analogously. Note that $U_{p^{*}}^{*}\left(R_{p^{*}}^{i}\right) \neq 1$, otherwise $U^{*} \equiv 1$, and we have a contradiction to the uniqueness of the initial value problem. Thus, for any sufficiently small $\bar{\varepsilon}>0$, we obtain

$$
U_{p^{*}}^{*}\left(R_{p^{*}}^{i}-\bar{\varepsilon}\right)>U_{p^{*}}^{*}\left(R_{p^{*}}^{i}\right) \quad \text { and } \quad U_{p^{*}}^{*}\left(R_{p^{*}}^{i}+\bar{\varepsilon}\right)>U_{p^{*}}^{*}\left(R_{p^{*}}^{i}\right) .
$$

Then (2.26) yields that, for $p$ sufficiently close to $p^{*}$, there exists a local minimizer $q_{p}$ of $U_{p}^{*}$ in $\left(R_{p^{*}}^{i}-\bar{\varepsilon}, R_{p^{*}}^{i}+\bar{\varepsilon}\right)$. Since $\bar{\varepsilon}>0$ was arbitrary, for each $p$ close to $p^{*}$, there is a local minimizer $q_{p}$ of $\left(U_{p}^{*}\right)^{\prime}\left(q_{p}\right)=0$ such that

$$
\lim _{p \rightarrow p^{*}} q_{p}=R_{p^{*}}^{i}
$$

On the other hand, if there exists a sequence $\left(p_{n}\right)_{n \in \mathbb{N}}$ such that $p_{n} \rightarrow p^{*}$ and $\left(q_{p_{n}}\right)_{n \in \mathbb{N}}$ converges to $R^{*}$, then by (2.26), one has $\left(U_{p^{*}}^{*}\right)^{\prime}\left(R^{*}\right)=0$. Equivalently $R^{*}=R_{p^{*}}^{j}$ for some $j$. Thus, we proved that the critical points of $U_{p}^{*}$ concentrate around critical points of $U_{p^{*}}^{*}$ and in arbitrary small neighborhood of $R_{p^{*}}^{i}$ there is a critical point of $U_{p}^{*}$.

To finish the proof, we show that in a small neighborhood of $R_{p^{*}}^{j}$, there exists at most one critical point of $U_{p}^{*}$. For a contradiction, assume that there exists a sequence $\left(p_{n}\right)_{n \in \mathbb{N}}$ such that $p_{n} \rightarrow p^{*}$ and both sequences $\left(q_{p_{n}}\right)_{n \in \mathbb{N}},\left(q_{p_{n}}^{\prime}\right)_{n \in \mathbb{N}}$ converge to $q^{*}$. Then by the mean value theorem, there exists $s_{p_{n}}$ between $q_{p_{n}}$ and $q_{p_{n}}^{\prime}$ such that $\left(U_{p^{*}}^{*}\right)^{\prime \prime}\left(s_{p_{n}}\right)=0$. By passing to the limit, one has $\left(U_{p^{*}}^{*}\right)^{\prime}\left(q^{*}\right)=\left(U_{p^{*}}^{*}\right)^{\prime \prime}\left(q^{*}\right)=0$, a contradiction to the fact that every critical point is either strict minimizer or strict maximizer (otherwise by the uniqueness of solutions to initial value problems, $U_{p^{*}}^{*}$ is constant).

Overall, we proved that in each neighborhood of $R_{p^{*}}^{i}$, there exists exactly one critical point of $U_{\lambda}^{*}$ and the proof is finished.

We are now in position to prove Theorem 1.4.

Proof of Theorem 1.4. By assumptions, we know that, for any $i \geq \tilde{i}, R_{\tilde{p}}^{i}>R$. On the other hand, by Proposition 2.6, for any $i \in \mathbb{N}, \lim _{p \rightarrow \infty} R_{p}^{i}<R$. Since the function $p \rightarrow R_{i}^{p}$, for any $i \in \mathbb{N}$, is continuous by Proposition 2.8, we deduce that there exists $p^{i}>\tilde{p}$ such that $R_{p^{i}}^{i}=R$. This concludes the proof.

\section{Proof of Proposition 1.6}

First, we show that $U_{p^{i}}^{*}$ has a finite (resp. infinite) Morse index provided that $p^{i}>p_{J L}$ (resp. $\left.2^{*}-1<p^{i}<p_{J L}\right)$, i.e. we prove Proposition 1.6. 
Proof of Proposition 1.6. Fix $p:=p^{i}>p_{J L}$. Then, for sufficiently small $\varepsilon_{0}>0$, one has

$$
p \theta(N-2-\theta)<\left(1-\varepsilon_{0}\right) \frac{(N-2)^{2}}{4} .
$$

Due to boundary conditions in (1.6), there exists $r_{0} \in(0,1)$ such that, for any $r \in\left(0, r_{0}\right)$,

$$
p\left(U_{p}^{*}\right)^{p-1}(r)-1 \leq p\left(U_{p}^{*}\right)^{p-1}(r) \leq \frac{p \theta(N-2-\theta)}{r^{2}}\left(1+\varepsilon_{0}\right) \leq \frac{(N-2)^{2}}{4 r^{2}}\left(1-\varepsilon_{0}^{2}\right) .
$$

Let $\chi_{0} \in C^{1}\left(\mathbb{R}^{N}\right)$ be a cut-off function such that $\chi_{0}(r)=\left\{\begin{array}{ll}1, & \text { if } r \in\left(0, r_{0} / 2\right) \\ 0, & \text { if } r>r_{0}\end{array}\right.$, and let $\chi_{1}=1-\chi_{0}$. We take $\phi \in H_{\text {rad }}^{1}\left(B_{R}(0)\right)$ such that $\phi^{\prime}(R)=0$. Then we have, thanks to the Hardy inequality,

$$
\begin{aligned}
\mathcal{J}(\phi) & =\int_{B_{R}(0)}\left(|\nabla \phi|^{2}-\left(p\left(U_{p}^{*}\right)^{p-1}(r)-1\right) \phi^{2} d x\right. \\
& =\int_{B_{R}(0)}\left(|\nabla \phi|^{2}-\left(\chi_{0}+\chi_{1}\right)\left(p\left(U_{p}^{*}\right)^{p-1}(r)-1\right) \phi^{2} d x\right. \\
& \geq \int_{B_{R}(0)}\left(\left(1-\varepsilon_{0}^{2}\right)|\nabla \phi|^{2}-\chi_{0} \frac{(N-2)^{2}}{4 r^{2}}\left(1-\varepsilon_{0}^{2}\right) \phi^{2} d x\right. \\
& +\int_{B_{R}(0)}\left(\varepsilon_{0}^{2}|\nabla \phi|^{2}-\chi_{1}\left(p\left(U_{p}^{*}\right)^{p-1}(r)-1\right) \phi^{2} d x\right. \\
& \geq \int_{B_{R}(0)}\left(\varepsilon_{0}^{2}|\nabla \phi|^{2}-\chi_{1}\left(p\left(U_{p}^{*}\right)^{p-1}(r)-1\right) \phi^{2} d x .\right.
\end{aligned}
$$

Since $\left.\mid p U_{p}^{*}\right)^{p-1}(r)-1 \mid \leq C$, for $r \in\left(r_{0} / 2, R\right)$, and the operator $-\varepsilon_{0}^{2} \Delta-\chi_{1}\left(p\left(U_{p}^{*}\right)^{p-1}(r)-\right.$ 1) with Neumann boundary condition has a finite number of negative eigenvalues, we conclude that $m\left(U_{p}^{*}\right)<\infty$.

Next assume that $2^{*}-1<p<p_{J L}$. As above, using boundary condition in (1.6), one has that, for some small $\varepsilon_{0}>0$, there exists $r_{0}$ such that, for all $r \in\left(0, r_{0}\right)$,

$$
p\left(U_{p}^{*}\right)^{p-1}(r)-1 \geq\left(\frac{(N-2)^{2}}{4}+\varepsilon_{0}^{2}\right) \frac{1}{r^{2}} .
$$

Next, we define $f_{j}(r)=f(r) \tilde{\chi}_{j}(r)$, where

$$
\tilde{\chi}_{j}(r)=\left\{\begin{array}{ll}
1, & \text { if } r \in\left[r_{j+1}, r_{j}\right], \\
0, & \text { elsewhere }
\end{array} \quad r_{j}=e^{-2 \pi j / \varepsilon_{0}}\right.
$$

and $f(r)=r^{-(N-2) / 2} \sin \left(\varepsilon_{0} \log r / 2\right)$. Notice that $f_{j}$ and $f_{k}$ have disjoint supports for $j \neq k$, and therefore they are linearly independent. Moreover, $f_{j}$ is a solution of

$$
-f_{j}^{\prime \prime}-\frac{N-1}{r} f_{j}^{\prime}-\left(\frac{(N-2)^{2}}{4}+\frac{\varepsilon_{0}^{2}}{4}\right) \frac{1}{r^{2}} f_{j}=0, \quad r \in\left(r_{j+1}, r_{j}\right) .
$$


Since $f_{j}\left(r_{j}\right)=f_{j}\left(r_{j+1}\right)=0$ we have that $f_{j} \in W^{1,2}((0, \infty))$ and by $(3.1)$

$$
\mathcal{J}\left(f_{j}\right) \geq \int_{r_{j+1}}^{r_{j}}\left(\left|f_{j}^{\prime}\right|^{2}-\left(\frac{(N-2)^{2}}{4}+\varepsilon_{0}^{2}\right) \frac{1}{r^{2}} f_{j}^{2}\right) r^{N-1} d r=-\frac{3}{4} \varepsilon_{0}^{2} \int_{r_{j+1}}^{r_{j}} \frac{1}{r^{2}} f_{j}^{2} d x<0 .
$$

Thus the Morse index of $U_{p}^{*}$ is infinite.

\section{References}

1. Ao, W., Chan, H., Wei, J.: Boundary concentrations on segments for the Lin-Ni-Takagi problem. Ann. Sc. Norm. Super. Pisa Cl. Sci. (5) 18(2), 653-696 (2018)

2. Ao, W., Musso, M., Wei, J.: On spikes concentrating on line-segments to a semilinear Neumann problem. J. Differ. Equ. 251(4-5), 881-901 (2011)

3. Bonheure, D., Casteras, J.-B., Földes, J.: Singular radial solutions for the Keller-Segel equation in high dimension. J. Math. Pures Appl. 134(9), 204-254 (2020)

4. Bonheure, D., Grossi, M., Noris, B., Terracini, S.: Multilayer radial solutions for a supercritical neumann problem. J. Differ. Equ. 261(1), 455-504 (2016)

5. Bonheure, D., Grumiau, C., Troestler, C.: Multiple radial positive solutions of semilinear elliptic problems with Neumann boundary conditions. Nonlinear Anal. 147, 236-273 (2016)

6. Budd, C., Norbury, J.: Semilinear elliptic equations and supercritical growth. J. Differ. Equ. 68(2), 169197 (1987)

7. Dancer, E.N., Yan, S.: Multipeak solutions for a singularly perturbed Neumann problem. Pac. J. Math. 189(2), 241-262 (1999)

8. del Pino, M., Mahmoudi, F., Musso, M.: Bubbling on boundary submanifolds for the Lin-Ni-Takagi problem at higher critical exponents. J. Eur. Math. Soc. (JEMS) 16(8), 1687-1748 (2014)

9. del Pino, M., Musso, M., Pistoia, A.: Super-critical boundary bubbling in a semilinear Neumann problem. Ann. Inst. H. Poincaré Anal. Non Linéaire 22(1), 45-82 (2005)

10. Dolbeault, J., Flores, I.: Geometry of phase space and solutions of semilinear elliptic equations in a ball. Trans. Am. Math. Soc. 359(9), 4073-4087 (2007)

11. Dupaigne, L.: Stable solutions of elliptic partial differential equations. Monographs and Surveys in Pure and Applied Mathematics, vol. 143. Chapman \& Hall/CRC, Boca Raton, FL (2011)

12. Esposito, P.: Estimations à l'intérieur pour un problème elliptique semi-linéaire avec non-linéarité critique. Ann. Inst. H. Poincaré Anal. Non Linéaire 24(4), 629-644 (2007)

13. Gel'fand, I.M.: Some problems in the theory of quasilinear equations. Am. Math. Soc. Transl. 2(29), 295-381 (1963)

14. Gierer, A., Meinhardt, H.: A theory of biological pattern formation. Biol. Cybern. 12, 30-39 (1972)

15. Guo, Z., Wei, J.: Global solution branch and Morse index estimates of a semilinear elliptic equation with super-critical exponent. Trans. Am. Math. Soc. 363(9), 4777-4799 (2011)

16. Joseph, D.D., Lundgren, T.S.: Quasilinear Dirichlet problems driven by positive sources. Arch. Ration. Mech. Anal. 49, 241-269 (1972/73)

17. Lee, Y., Seok, J.: Multiple interior and boundary peak solutions to singularly perturbed nonlinear Neumann problems under the Berestycki-Lions condition. Math. Ann. 367(1-2), 881-928 (2017)

18. Lin, C.-S., Ni, W.-M., Takagi, I.: Large amplitude stationary solutions to a chemotaxis system. J. Differ. Equ. 72(1), 1-27 (1988)

19. Lin, C.S., Ni, W.-M.: On the diffusion coefficient of a semilinear Neumann problem. Calculus of variations and partial differential equations (Trento, 1986), pp. 160-174 (1988)

20. Malchiodi, A.: Concentrating solutions of some singularly perturbed elliptic equations. Front. Math. China 3(2), 239-252 (2008)

21. Merle, F., Peletier, L.A.: Positive solutions of elliptic equations involving supercritical growth. Proc. R. Soc. Edinb. Sect. A 118(1-2), 49-62 (1991)

22. Miyamoto, Y.: Classification of bifurcation diagrams for elliptic equations with exponential growth in a ball. Ann. Mat. Pura Appl. (4) 194(4), 931-952 (2015)

23. Miyamoto, Y.: Structure of the positive radial solutions for the supercritical Neumann problem $\varepsilon^{2} \Delta u-$ $u+u^{p}=0$ in a ball. J. Math. Sci. Univ. Tokyo 22(3), 685-739 (2015)

24. Ni, W.-M., Takagi, I.: On the shape of least-energy solutions to a semilinear Neumann problem. Commun. Pure Appl. Math. 44(7), 819-851 (1991)

25. Ni, W.-M., Takagi, I.: Locating the peaks of least-energy solutions to a semilinear Neumann problem. Duke Math. J. 70(2), 247-281 (1993) 
26. Rey, O.: An elliptic Neumann problem with critical nonlinearity in three-dimensional domains. Commun. Contemp. Math. 1(3), 405-449 (1999)

27. Rey, O.: The question of interior blow-up-points for an elliptic Neumann problem: the critical case. J. Math. Pures Appl. (9) 81(7), 655-696 (2002)

28. Rey, O., Wei, J.: Blowing up solutions for an elliptic Neumann problem with sub- or supercritical nonlinearity. I. $N=3$. J. Funct. Anal. 212(2), 472-499 (2004)

29. Wei, J.: Existence and stability of spikes for the Gierer-Meinhardt system. In: Handbook of Differential Equations: Stationary Partial Differential Equations, vol. V, pp. 487-585 (2008)

Publisher's Note Springer Nature remains neutral with regard to jurisdictional claims in published maps and institutional affiliations. 